\title{
Platform for moisture content determination of fine-grained soil
}

Nisat Sarwar Shetu BSC

Associate Member, The Institute of Engineers, Dhaka, Bangladesh
Md Abdullah Masum BSC

Graduate Student Member, Institution of Electrical and Electronics Engineers, New York, NY, USA

Determination of moisture content of soil is crucial and must be performed as frequently as necessary to ensure the quality of construction work. In order to address the limitations and inaccuracies of existing methods, a new concept has been implemented. An artificial neural network and state-of-the-art electronic circuitry are embedded to realise an automatic measurement system based on a low-cost computing platform. Also, to facilitate user interaction with the system, a sophisticated graphical user interface was created. This system, from both a hardware and a software perspective, brings new ideas not only to the moisture content determination test but also to the geotechnical laboratory and field instrumentation in terms of accuracy, automation and machine intelligence. This work shows that hardware and software embedded in test equipment can automate the test procedures in geotechnical engineering, using an appropriate sensor and a data acquisition and processing routine. This paper presents the technical details of the system set-up, hardware and software development, workflow and system validation for the determination of moisture content of fine-grained soil.

\section{Notation}

$N \quad$ number of soil samples

$R^{2} \quad$ highest regression coefficient

$W_{\mathrm{c}} \quad$ weight of the container

$W_{\text {dc }} \quad$ weight of a dry soil sample with container

$W_{\text {wc }} \quad$ weight of a wet soil sample with container

$e_{j}(n) \quad$ error for neuron $j$ at the $n$th iteration

$w \quad$ moisture content of soil

$\Delta w_{j i} \quad$ synaptic weight correction connecting neuron $i$ to neuron $j$

\section{Introduction}

Moisture content is one of the most significant factors for evaluating the strength of soil. Planning of any structure and its foundation requires taking into consideration the strength of the underlying soil, which is determined from test results and is highly dependent on moisture content (Carlos et al., 2001). However, without knowing the moisture content, the optimal strength of soil cannot be ensured reliably (Vanapalli et al., 1996) in the construction site by merely applying the so-called soil compaction method (Budhu, 2011) and soil stabilisation technique (Garber and Hoel, 2008). Moisture content can be determined in accordance with various international standards (NDOR, 2012). According to ASTM D 2216, the weight of a suitable aluminium can with some soil sample is determined and then the can is heated in a conventional oven at $110^{\circ} \mathrm{C}$ for $24 \mathrm{~h}$. After $24 \mathrm{~h}$ of drying, the can with dry sample is weighed again and, from the difference between this measurement and the previous one, moisture content is estimated. In this procedure, a small amount of energy is actually used in the drying process and the remaining significant portion is lost. Therefore, this is not an energy-efficient and environmentally friendly method.
Moreover, spending $24 \mathrm{~h}$ on drying time may have a detrimental impact on the construction process if evaluation of the soil strength is necessary during this time and in subsequent phases. Recently, a slightly faster microwave-oven-based procedure has been introduced. In this approach, the first cycle of the drying process of a soil sample continues for $3 \mathrm{~min}$ under a constant microwave power and after that both weighing and mixing of the sample are conducted. For subsequent cycles, drying time is reduced to $1 \mathrm{~min}$ and both weighing and mixing are repeated until two consecutive weights differ only by $0 \cdot 1 \%$ or less from the initial weight of the soil taken (Hagerty et al., 1990). This microwave-based moisture content determination is faster than the conventional oven-based method, but it requires frequent manual interventions and there is no automatic control for data acquisition and processing. As a consequence, inaccuracies may arise from human factors. Some variations of this test method can be found in the literature (Chung and Ho, 2008), but these methods also suffer from the same frequent manual interventions and lack of automatic control for data acquisition and processing. Another approach for the determination of moisture content of soil mainly used in irrigation purposes is the neutron scattering method (Van et al., 1961). In this method, radiation is the key to measuring the volumetric moisture content and hence bulk specific gravity must be considered. However, this approach suffers from high instrumental cost, radiation hazard and an error of up to 15 per cent (Zazueta and Xin, 1994), which make it inappropriate in this particular context. In the last few years, artificial neural networks (ANNs) have played an important role in such fields as aerospace, automotive, telecommunications and transportation (Chaturvedi et al., 2002; Demuth and Beale, 2002; Smith and Demetsky, 1994). In geotechnical engineering, ANNs have been used successfully in the prediction of lateral load capacity 
of piles (Das and Basudhar, 2006), in the modeling of maximum dry density and optimum moisture content of soil (Alavi et al., 2010) and in determining the hydraulic conductivity of compacted finegrained soils (Erzin et al., 2009). It has been shown that ANNs can be used successfully for the prediction of moisture content of finegrained soil in the shortest time possible (Shetu and Masum, 2012), but this approach suffers from several limitations. This system is intended only for moisture content determination of soil using proprietary MatLab software (MathWorks, 2014), data acquisition (DAQ) module and Windows-based PC, which incurs a significant cost. Moreover, due to its proprietary nature, the communication module and associated protocols are not suitable for other types of sensors for performing various tests. With the advancement in modern computing technology, ultra-low-cost, high-performance, open-source computers have become available, which eliminate the need for high-cost proprietary PCs, modules and software. This fact motivated the authors build an open-source, low-cost system that generalises the concept of a unified framework that is extremely reconfigurable to cope with various sensor architectures necessary for performing many types of tests using a simplified open-source programming model. In addition, the proposed system should be small, efficient, powerful, intelligent, terse, modular, pragmatic and coherent in its design.

In this paper, the authors propose a novel approach for the determination of moisture content of subgrade soil using a custom-built hardware system. The notable components are a \$35 open-source, Linux-based, credit card-sized Raspberry Pi computer (Raspberry Pi Foundation, UK); a microwave oven; and a PIC18LF4550 microcontroller (Microchip Technology, 2007; Rafiquzzaman, 2011)-based customisable electronic interfacing circuitry. Although the primary purpose of the Raspberry Pi computer is to promote programming for schoolchildren (CellanJones, 2011; Halfacree and Upton, 2012), it has attracted millions of computer enthusiasts around the globe since its inception because of its high-performance, ARM-based, $700 \mathrm{MHz}$ soft CPU (central processing unit) core as well as its wide range of connectivity options to other hardware peripherals such as sensors, camera modules and many more. All these components together make the proposed system fully automatic and intelligent enough to avoid every manual interaction and the possible inaccuracies they may cause. The fully trained embedded neural network offers intelligence in the form of predictive capability, which can provide the result in advance without going through the entire length of the test period. Experiments show that time and power consumption are lowered by about $50 \%$ compared with current fast microwaveoven-based methods. In addition, the developed electronic module can be battery operated, portable and easily reconfigured for use with other sensors (pressure, temperature, humidity etc.) for various tests in civil engineering. Moreover, this system allows remote log-in facility using the secure shell (SSH) internet protocol, which facilitates the conducting of tests in the absence of any expert technician at the test location. The remaining sections of this paper are organised as follows. The next section presents some background theories; a detailed explanation of the system set-up and experimental procedure is outlined in the subsequent sections. After that, the test results with discussions and, finally, a conclusion with possible future directions are presented, respectively, in the last two sections.

\section{Background theory}

Moisture content of soil

Soil is composed of solid particles, water and air. The ratio of the weight of water present in soil to the weight of solids is called the moisture content (Budhu, 2011) and is formulated as follows

1. $\quad w=\left(W_{\mathrm{wc}}-W_{\mathrm{dc}}\right) /\left(W_{\mathrm{dc}}-W_{\mathrm{c}}\right) \times 100 \%$

where $W_{\mathrm{wc}}, W_{\mathrm{dc}}$ are, respectively, the weights of wet and dry samples of soil with container, and $W_{\mathrm{c}}$ is the weight of the container.

\section{Microwave heating fundamentals}

A microwave occupies the frequency range of approximately $0 \cdot 3$ to $300 \mathrm{GHz}$ in the electromagnetic spectrum. The most common commercial microwave generator, magnetron, is found in microwave ovens and has a rated frequency of $2 \cdot 45 \mathrm{GHz}$. This frequency is very close to the natural frequency of water molecules. Due to resonance, at this frequency vigorous vibration of water molecules present in soil samples causes intermolecular frictions that, in turn, generate heat (Cann, 2011). As a result, the temperature of the soil mass increases gradually toward the boiling point of water and, eventually, evaporation occurs, thus allowing the sample to dry in a comparatively short time. According to ASTM D 4643-08, in the determination process of soil moisture content, microwave oven drying gives rapid results. As microwaves have to penetrate into the soil, attenuation of microwave power occurs in every consecutive layer (Lord et al., 1979). Due to the requirement of microwave penetration into a soil sample, the dimensions should not be too coarse.

\section{Material and methods}

Set-up

The full system consists of a microwave oven; an electronic balance; an open-source, Linux-based, credit card-sized computer (Raspberry Pi); and a PIC18LF4550 microcontroller. Notable components and the signalling control mechanisms between them, from both software and hardware perspectives, are shown in Figure 1. The system model consists of some sensors (i.e., temperature, humidity, flow, strain etc.) that collect measurements and send data to a signal-conditioning unit, whose role is to make signals suitable (i.e., noise reduced) for processing in the microcontroller. The microcontroller acts as an interface between the signal-conditioning unit and the main computer, which runs the developed logic and issues the commands to perform the test. This model was implemented for the moisture content determination and is shown in Figure 2(a). A Panasonic microwave oven (NNSD691S) of dimensions $525 \times 401 \times 310 \mathrm{~mm}$ was used. It has 


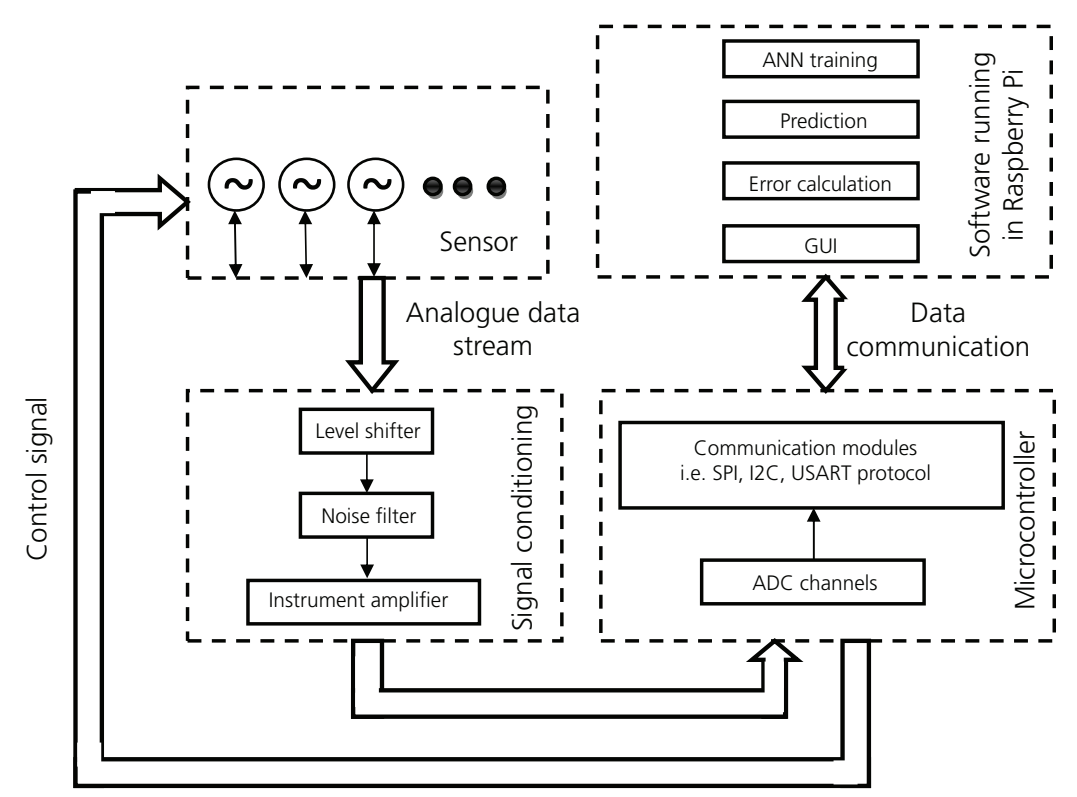

Figure 1. Block diagram representation of the overall system

a rated input power of $700 \mathrm{~W}$, which meets the power requirement of ASTM D4643-08. An electronic balance (Model CB-V Electronic Balance) having a precision of $0.01 \mathrm{~g}$ and a capacity of $2000 \mathrm{~g}$ was employed for measuring the weights of samples. The PIC18LF4550 microcontroller was programmed with the authors' firmware (developed in the $\mathrm{C}$ programming language) to realise a highly customisable DAQ module, which can be interfaced with up to 13 analogue sensors using its analogue-to-digital converter channels (each of 10-bit resolution) simultaneously. This microcontroller was also configured as a sophisticated interfacing module for bi-directional communication between temperature and weight sensors, computer and microwave oven. This module is set to sample weight and temperature signals up to 1000000 samples/s and can also interpret the commands issued by the developed graphical user interface (GUI) to the microwave oven through an opto-coupler (MOC5007). The opto-coupler isolates the high-voltage $(230 \mathrm{~V})$ side of the oven from the low-voltage $(5 \mathrm{~V})$ side of the computer and interfacing module. The GUI was designed using a widely used user interface design software, Qt Designer, to act as an interface for the human operator to run the experiment successfully (Harwani, 2012). A screenshot of the GUI is shown in Figure 2(b). A hole was cut out of the bottom of the microwave oven and it was attached to a four-legged stand so that the balance could be placed in between the stand and the oven. These works were done to facilitate the continuous recording of the weight signal from the electronic balance as voltage using interfacing circuitry and passing to the Raspberry Pi computer for analysis. A temperature sensor was placed in the microwave oven to protect the oven from extra heat in case of the sample being too dry or having some mineral that may react with microwaves. If the temperature increases to more than $110^{\circ} \mathrm{C}$ (which is the maximum acceptable temperature stated in ASTM D 2216), then the absorbed water is driven off from the soil mass, which is not accounted for in moisture content determination. In this case, the computer automatically stops the oven by analysing the data from the temperature sensor to avoid any accidental damage. All the communications between the microcontroller and Raspberry Pi computer are done using the I2C and UART communication protocol. This custom-built electronic circuitry, including the computer, cost less than $\$ 100$. Moreover, the software was developed using the open-source Python 2.7.3 scripting language, which is free of charge (Python, 2014).

\section{Procedure}

\section{Sample preparation}

A total of 180 soil samples, each weighing approximately $100 \mathrm{~g}$, was collected in a $250-\mathrm{mL}$ tempered glass container successively following a criterion stated in ASTM D 4643-00 - that is, that sample weight should be 100-200 g. Another ten sample pairs of the same type were also taken for separate microwave oven and conventional oven experiments. A tempered glass container was chosen because of its microwave and thermal resistance, and its shape favours the effective vaporisation of water. The soil samples were collected from a road construction site near Majura Road, Canberra, Australia; they were classified as fine grained according to the Unified Soil Classification System (USCS) (ASTM, 2011).

\section{Operating principle}

The proposed system is operated algorithmically by software (written in Python), which incorporates a custom-built GUI (shown in Figure 2(b)) and can be worked in two distinct modes: unsupervised and supervised. 
Platform for moisture content

determination of fine-grained soil

Shetu and Masum

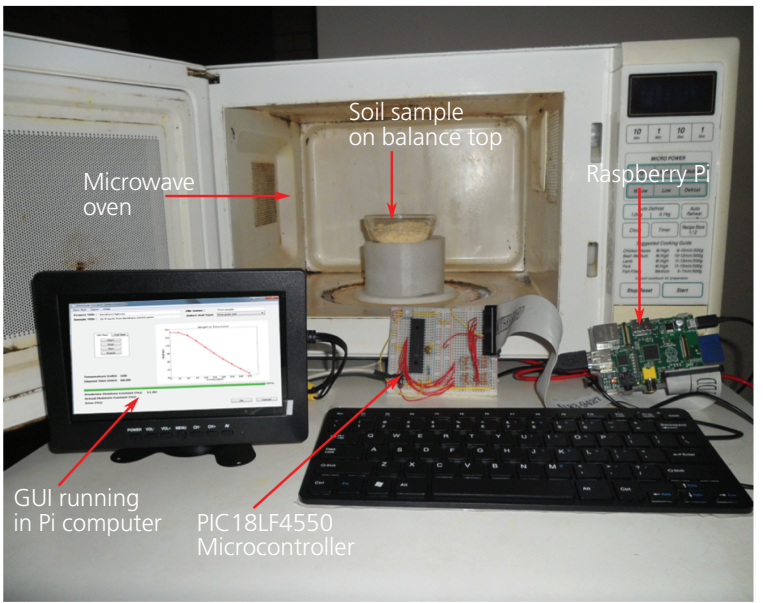

(a)

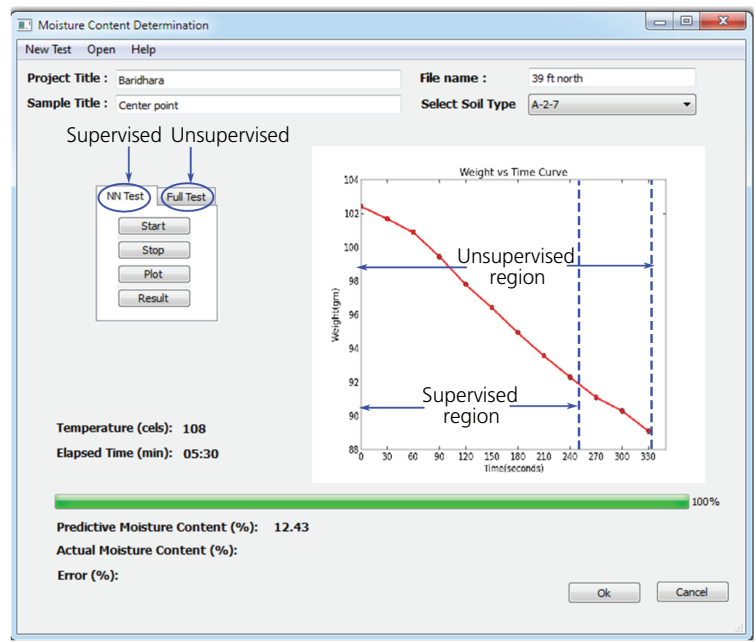

(b)

Figure 2. (a) Experimental set-up; (b) developed GUI with a plotted sample dataset

In unsupervised mode, the data acquisition and storage are performed automatically in computer flash memory. The whole test takes about 11-15 min for each sample depending on its moisture content. At the beginning of the experiment, the oven and the clock were turned on and the computer started storing the weights of the sample at $30 \mathrm{~s}$ intervals as it progressively lost moisture. When two successive weights differed by $0 \cdot 1 \%$ or less, the process was automatically terminated and moisture content was calculated using Equation 1.

To enable the system operation in supervised mode, a trained neural network was incorporated in the developed software. For training the neural network, the same experiment as performed in unsupervised mode was repeated for 179 soil samples. The purpose of these soil samples was to train the neural network and evaluate its accuracy compared to the ground truth conventional oven-based method. Subsequently, this trained neural network can be used to predict the moisture content without performing the full test, thus reducing the test time by at least $50 \%$ compared with the unsupervised mode.

\section{Supervised mode: training the neural network}

For this work, a two-layer (i.e., a sigmoid hidden layer and a linear output layer) supervised feedforward back-propagation neural network was developed. This network architecture was chosen because one hidden layer is sufficiently capable of simulating a function representing a continuous relationship between input and output (Heaton, 2008). From several available optimisation algorithms, the Levenberg-Marquardt back-propagation algorithm was nominated to optimise the errors due to its robustness, efficiency and ability to handle nonlinear problems (MathWorks, 2014). A total of 180 sample datasets was chosen because the network should be capable of predicting the unknown variables (total number of network weights and biases) very reliably. Batch mode training was chosen to aim for higher accuracy. An initial 12 records (each dataset contained 22 to 30 records, which was equivalent to $11-15 \mathrm{~min}$ ) from 180 datasets of soil samples were considered for training the network, while the training sets can be represented as

$$
\text { 2. } \quad \mathfrak{I}=\left\{\left(x_{p}, d_{p}\right)\right\}_{p=1}^{N}
$$

where $N, x_{p}$ and $d_{p}$ are the number of soil samples, a set of 12 successive weights of soil sample and the associated water content, respectively. Figure 3(a) depicts the propagation path of signal flow of output layer neuron $j$, which was fed by the outputs of the previous layer's neurons. The induced local field $v_{j}(n)$ is the sum of weighted input nodes of neuron $j$ at the $n$th iteration and can be expressed as

3.

$$
v_{j}(n)=\sum_{i=0}^{m}\left[w_{j i}(n) y_{i}(n)\right]
$$

The output of neuron $j$ is calculated by

$$
\text { 4. } \quad y_{j}(n)=f_{j}\left[v_{j}(n)\right]
$$

$f_{j}$ is the activation function of neuron $j$. The error for neuron $j$ at the $n$th iteration is

$$
\text { 5. } e_{j}(n)=d_{j}-y_{j}(n)
$$

Then the corrected weight is

$$
\text { 6. } w_{j i}(n+1)=w_{j i}(n)-\left[J(n)^{T} J+\mu I\right]^{-1} J(n) e_{j}(n)
$$

where $\mu$ is the learning-rate parameter and $J(n)$ is the Jacobian matrix calculated as 


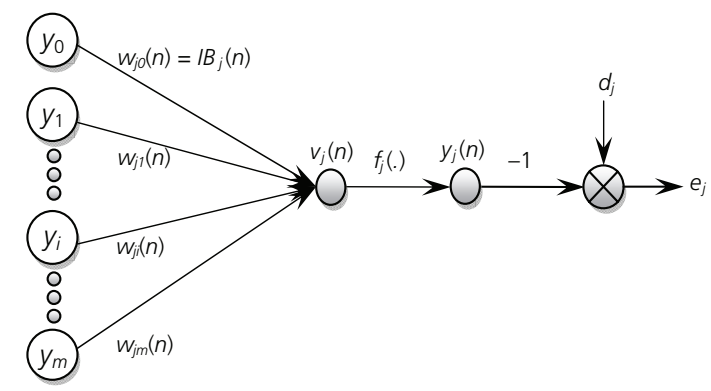

(a)

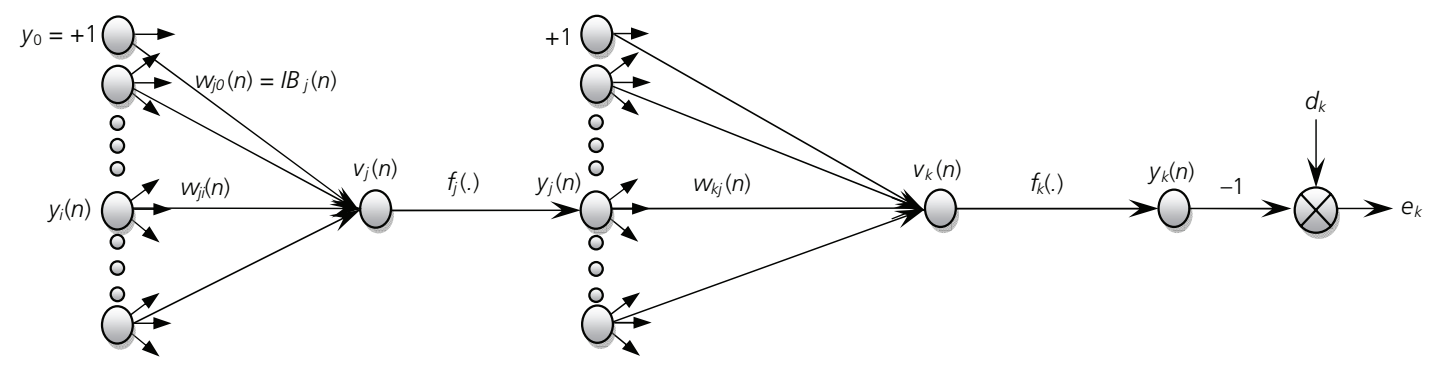

(b)

Figure 3. (a) Signal-flow diagram of an output neuron $j$;

(b) signal-flow diagram of an output neuron $k$ connected to hidden neuron $j$ (adapted from Haykin, 2005)

7. $\quad J(n)=\left[\begin{array}{cccc}\frac{\partial e_{1}(n)}{\partial w_{j 1}(n)} & \cdots & \frac{\partial e_{1}(n)}{\partial w_{j m}(n)} & \frac{\partial e_{1}(n)}{\partial w_{j 0}(n)} \\ \vdots & \ddots & \vdots & \vdots \\ \frac{\partial e_{N}(n)}{\partial w_{j 1}(n)} & \cdots & \frac{\partial e_{N}(n)}{\partial w_{j m}(n)} & \frac{\partial e_{N}(n)}{\partial w_{j 0}(n)}\end{array}\right]$

If neuron $j$ is in the hidden layer, there is no specified desired response for that neuron and error as well. Another network, shown in Figure 4, can be considered where neuron $j$ is in the hidden layer and connected to the output layer containing neuron $k$. The local gradient $\delta_{j}(n)$ for hidden neuron $j$ can be found as

$$
\begin{aligned}
\delta_{j}(n) & =-\frac{\partial \xi(n)}{\partial v_{j}(n)} \\
& =-\frac{\partial \xi(n)}{\partial y_{j}(n)} \frac{\partial y_{j}(n)}{\partial v_{j}(n)} \\
& =-\frac{\partial \xi(n)}{\partial y_{j}(n)} f_{j}^{\prime}\left[v_{j}(n)\right]
\end{aligned}
$$

In batch mode, $\xi(n)$ is the average error energy and is defined as
9. $\quad \xi(n)=\frac{1}{2 N} \sum_{p=1}^{N} \sum_{k \in C} e_{k}^{2}(n)$

$C$ is the number of neurons in the output layer. Now, differentiating Equation 9 with respect to the functional signal $y_{j}(n)$ yields

$$
\begin{aligned}
\frac{\partial \xi(n)}{\partial y_{j}(n)} & =\frac{1}{2 N} \sum_{p=1}^{N} \sum_{k} e_{k} \frac{\partial e_{k}(n)}{\partial y_{j}(n)} \\
& =\frac{1}{2 N} \sum_{p=1}^{N} \sum_{k} e_{k}(n) \frac{\partial e_{k}(n)}{\partial v_{k}(n)} \frac{\partial v_{k}(n)}{\partial y_{j}(n)}
\end{aligned}
$$

From Figure 4, it is easily noted that

$$
\begin{aligned}
e_{k}(n) & =d_{k}(n)-y_{k}(n) \\
& =d_{k}(n)-f_{k}\left[v_{k}(n)\right]
\end{aligned}
$$

11.

Thus

12. $\frac{\partial e_{k}(n)}{\partial v_{k}(n)}=-f k^{\prime}\left[v_{k}(n)\right]$ 


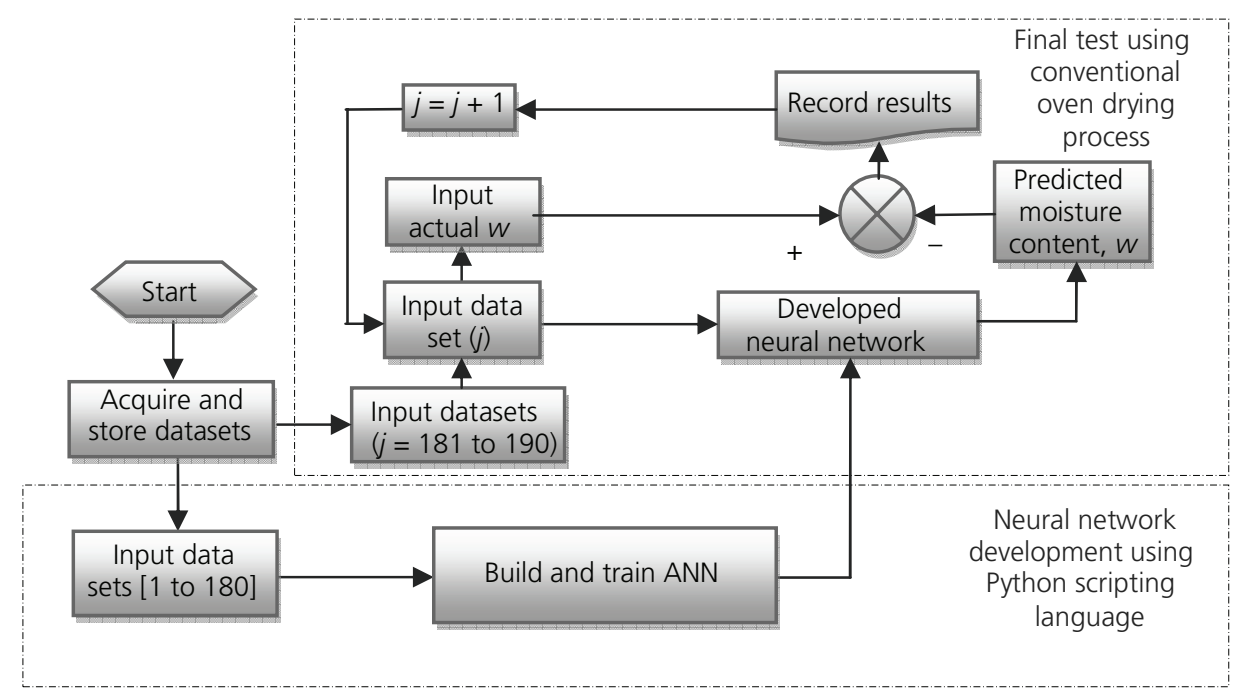

Figure 4. The algorithmic workflow of ANN development and error calculation

It is clearly seen in Figure 4 that the local induced field $v_{k}$ can be regarded as

13.

$$
v_{k}(n)=\sum_{j=0}^{m} w_{k j}(n) y_{j}(n)
$$

$m$ is the total number of inputs connected to neuron $k$. Differentiating Equation 13 with respect to $y_{j}(n)$ gives

14. $\frac{\partial v_{k}(n)}{\partial y_{j}(n)}=w_{k j}(n)$

Substituting Equations 12 and 14 in Equation 10 yields

15.

$$
\begin{aligned}
\frac{\partial \xi(n)}{\partial y_{j}(n)} & =-\frac{1}{2 N} \sum_{p=1}^{N} \sum_{k} e_{k}(n) f_{k}^{\prime}\left[v_{k}(n)\right] w_{k j}(n) \\
& =-\sum_{k} \delta_{k}(n) w_{k j}(n)
\end{aligned}
$$

$\delta_{k}(n)$ is the local gradient of neuron $k$. Using Equation 15 in Equation 8 gives the back-propagation formula for the local gradient as follows

16.

$$
\delta_{j}(n)=f_{j}^{\prime}\left[v_{j}(n)\right] \sum_{k} \delta_{k}(n) w_{k j}(n)
$$

The correction $\Delta w_{j i}$ applied to synaptic weight connecting neuron $i$ to neuron $j$ is defined as follows
17. $\Delta w_{j i}(n)=\mu \cdot \delta_{j}(n) \cdot y_{i}(n)$

Then new weight is found as

18. $w_{j i}(n+1)=w_{j i}(n)+\Delta w_{j i}(n)$

Equations 1-18 somewhat resemble those formulated by Haykin (2005). After the above steps, adjustment of weights continued until the lowest mean squared error (MSE) and the highest regression coefficient $\left(R^{2}\right)$ were reached. MSE and $R^{2}$ are formulated as follows

19. $\mathrm{MSE}=\frac{\sum_{p=1}^{N}\left(d_{p}-y_{p}\right)^{2}}{N}$

20. $\quad R^{2}=1-\frac{\sum_{p=1}^{N}\left(d_{p}-y_{p}\right)^{2}}{\sum_{p=1}^{N}\left(d_{p}-\overline{d_{p}}\right)^{2}}$

where $y_{p}$ and $\overline{d_{p}}$ are the calculated moisture content and the average of desired moisture content, respectively. The highest regression coefficient, $R^{2}=1$, indicates an exact relationship between predicted and actual moisture content, while the lowest value of $R^{2}=0$ means no relationship exists at all. The datasets were passed through the proposed algorithm, following the above equations implemented 
Platform for moisture content

determination of fine-grained soil

Shetu and Masum

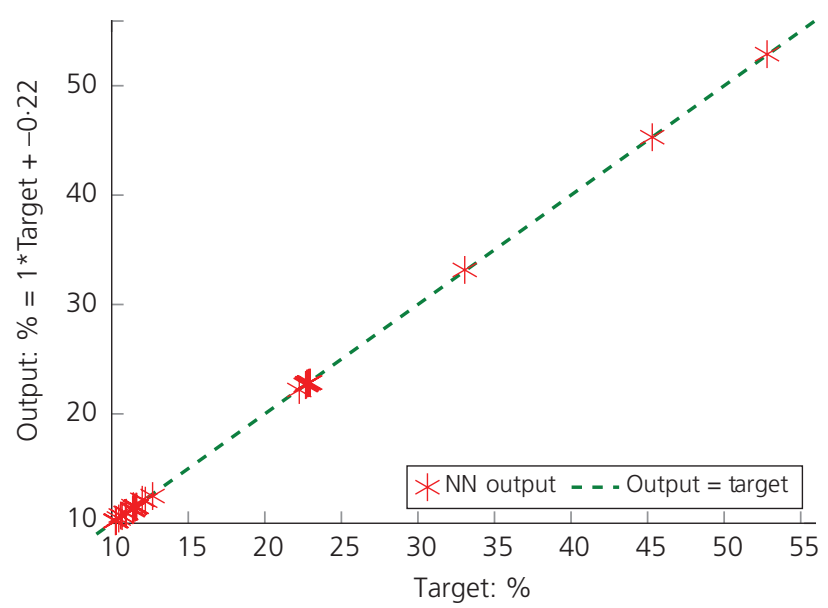

(a)

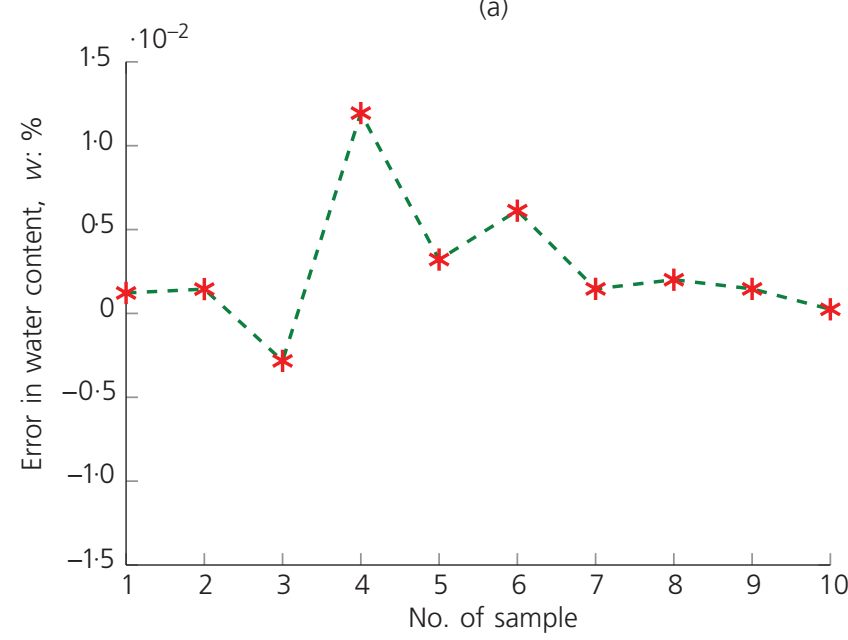

(b)

Figure 5. (a) Predicted water content plotted against actual water content for test datasets; (b) discrepancy between actual and predicted water content

using the MATLAB programming language, which performed the training, validation and preliminary testing of the ANN. As a rule of thumb, the optimum number of neurons in the hidden layer should be between two-thirds and twice the number of input layer elements (Heaton, 2008). For this experiment, the optimum number of neurons in the hidden layer was found to be 16 by trading off accuracy and computational burden.

Final test using microwave and conventional oven data The ultimate validation of this system was performed in real time using the last ten datasets (each dataset consisted of 12 records instead of the full 22 to 30 records) obtained from the microwave oven, which also had actual moisture content, obtained from conventional oven drying following the ASTM D 2216-10 standard. This reduced number of records corresponds to the reduction in time of about $50 \%$. Datasets acquired from microwave-oven testing were passed through the developed neural network. The outputs of the network were the predicted values of moisture contents, which were then compared with the moisture content of respective soil samples obtained from the conventional oven drying process. The whole algorithmic workflow for neural network development and validation is shown in Figure 4 as a flowchart. In this figure, development of the neural network was performed offline. The discrepancy between the actual moisture contents from the conventional oven drying process and the predicted moisture contents from ANN is shown in Figure 5(b). In practice, the system is intended for real-time prediction of moisture content in both modes - supervised and unsupervised.

\section{Results and discussion}

The performance of the developed network for test, training and validation datasets is shown in Figure 5(a), Figure 6(a) and Figure $6(\mathrm{~b})$, respectively. The data points represent the neural network

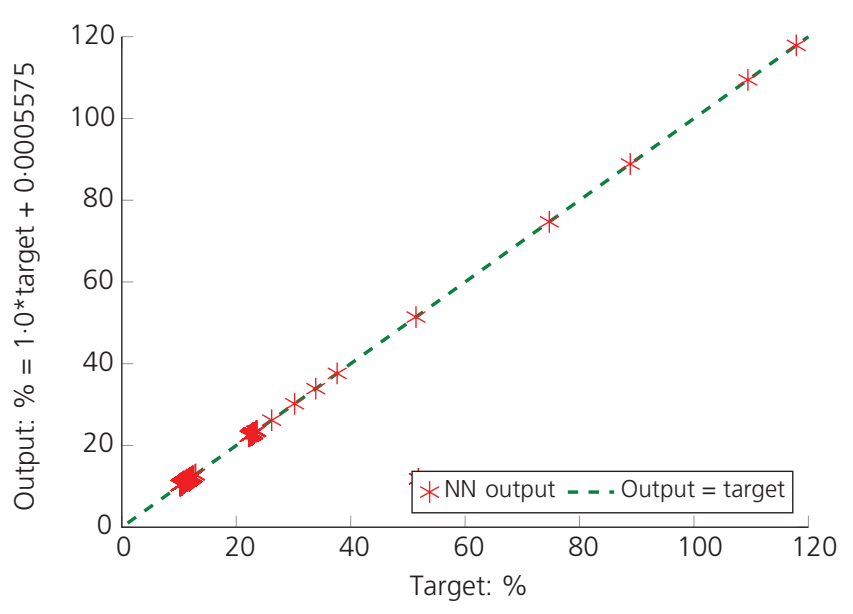

(a)

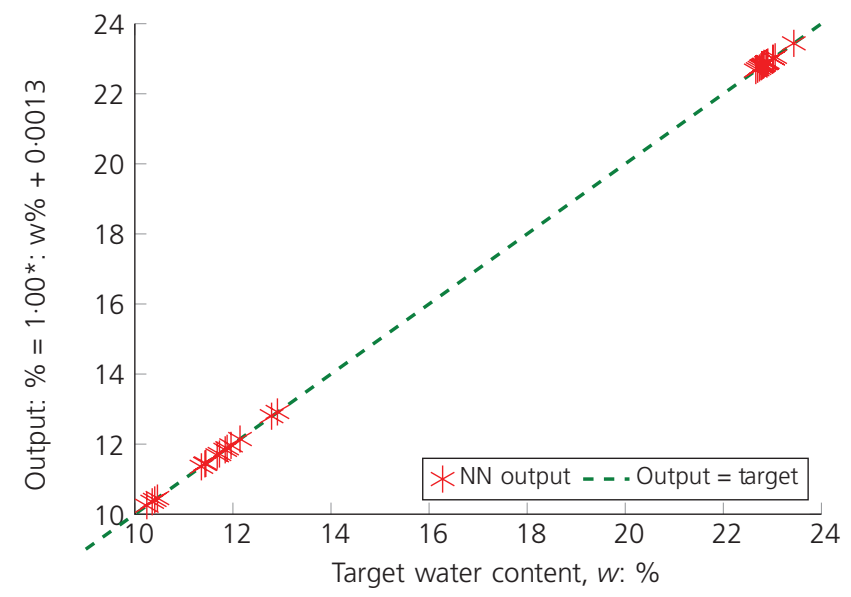

(b)

Figure 6. Predicted water content plotted against actual water content for (a) training datasets and (b) validation datasets 
outputs to the target moisture contents. Hence the linear dashed lines indicate that the predicted moisture contents are equal to the target moisture contents. The maximum MSE for different types of datasets was found to be 0.1361 and the regression coefficient $R^{2}$ to be $1 \cdot 0$ for all types of datasets. It is obvious from the relationship $R^{2}=1$ that there exists an exact linear relationship between the ANN outputs and targets. From these graphs, it is also evident that the trained network works precisely well with soil samples having moisture content of up to $123 \%$. Additionally, Figure 5(b) reveals the discrepancy between the actual moisture contents obtained from a conventional oven and the predicted values from ANN. It should be noted that the maximum difference was only $0.08 \%$, which can be overlooked considering the huge amount of time and energy saving (almost 50\% less than the existing microwave-oven-based method).

\section{Conclusions}

The outcomes of this work indicate that the soil drying process for moisture content determination can be realised efficiently using a group of algorithmic actions, which are performed in real time using a hardware and a software platform. The implemented system not only presents the precise results in the shortest time possible but also automates the whole test procedure using very low-cost, open-source electronic programmable hardware. Portability, robustness and large test range are among its attributes. Therefore, generalisation of the proposed system considering all types of soil in a geotechnical laboratory should be the ultimate goal for a future endeavour.

\section{REFERENCES}

Alavi A, Gandomi AH, Mollahassani A, Heshmati AA and Rashed A (2010) Modelling of maximum dry density and optimum moisture content of stabilized soil using artificial neural networks. Journal of Plant Nutrition and Soil Science 173(3): 368-379.

ASTM (2011) D 2487: Standard practice for classification of soils for engineering purposes 294 (Unified Soil Classification System). ASTM International, West Conshohocken, PA, USA.

Budhu M (2011) Soil Mechanics and Foundations. Wiley, Hoboken, NJ, USA.

Cann M (2011) Microwave Heating as a Tool for Sustainable Chemistry. CRC Press, Boca Raton, FL, USA.

Carlos MV, Luis HB and Jan WH (2001) Contribution of water content and bulk density to field soil penetration resistance as measured by a combined cone penetrometer-TDR probe. Soil and Tillage Research 60: 35-42.

Cellan-Jones R (2011) A 15 pound computer to inspire young programmers. $B B C$ News, 5 May.

Chaturvedi D, Chauhan R, and Kalra P (2002) Applications of generalised neural network for aircraft landing control system. Soft Computing 6(6): 441-448.

Chung PWK and Ho TYK (2008) Study on the Determination of Moisture Content of Soils by Microwave Oven Method. Civil
Engineering and Development Department, The Government of Hong Kong, PR China, Geo report no. 221.

Das SK and Basudhar PK (2006) Untrained lateral load capacity of piles in clay using artificial neural network. Computers and Geotechnics 33(8): 454-459.

Demuth $\mathrm{H}$ and Beale M (2002) Neural Network Toolbox for Use with MATLAB. The MathWorks, Inc., Natick, MA, USA.

Erzin Y, Gumaste SD, Gupta AK and Singh DN (2009) Artificial neural network (ANN) models for determining hydraulic conductivity of compacted fine-grained soils. Canadian Geotechnical Journal 46(8): 955-968.

Garber N and Hoel L (2008) Traffic \& Highway Engineering, 4th edition. Cengage Learning, Toronto, Canada.

Hagerty D, Ullrich C, and Denton M (1990) Microwave drying of soils. Geotechnical Testing Journal 13(2): 138-141.

Halfacree G and Upton E (2012) Raspberry Pi User Guide. Wiley, Hoboken, NJ, USA.

Harwani BM (2012) Introduction to Python Programming and Developing GUI Applications with PyQT. Course Technology, Cengage Learning, Independence, KY, USA.

Haykin S (2005) Neural Networks A Comprehensive Foundation, 2nd edition. Pearson Education, Singapore.

Heaton J (2008) Introduction to Neural Networks with C\#, 2nd edition. Heaton Research, Inc., Chesterfield, MO, USA.

Lord AE, Korner R and Reif J (1979) Determination of attenuation and penetration depth of microwaves in soil. Geotechnical Testing Journal 2(2): 403-409.

MathWorks (2014) Neural Network Toolbox. The MathWorks, Inc., Natick, MA, USA. See http://www.mathworks.com.au/ help/nnet/index.html (accessed 28/01/2014).

Microchip Technology (2007) 28/40/44-pin High Performance, Enhanced Flash USB Microcontrollers with nanoWatt Technology. Datasheet. Microchip Technology Inc., Chandler, AZ, USA.

NDOR (Nebraska Department of Roads) (2012) Geotechnical Policies and Procedures Manual. Nebraska Department of Roads, Lincoln, NE, USA.

Python (2014). Python Programming Language. Official Website. Python Software Foundation, Beaverton, OR, USA. See http://www.python.org/ (accessed 28/01/2014).

Rafiquzzaman M (2011) Microcontroller Theory and Applications with the PIC18F, 1st edition. Wiley, Hoboken, NJ, USA.

Shetu NS and Masum MA (2012) A novel approach for the determination of moisture content of soil using artificial neural network and automatic electronic control. Proceedings of Ground Engineering in a Changing World, the 11th AustraliaNew Zealand Conference on Geomechanics, Melbourne, Australia, pp. 517-522.

Smith B and Demetsky M (1994) Short-term traffic flow prediction models - a comparison of neural network and nonparametric regression approaches. Systems, man, and cybernetics. Humans, Information and Technology 2: 1706-1709. 
Van BC, Nielsen D and Davidson J (1961) Calibration and characteristics of two neutron moisture probes. Soil Science Society of America Proceedings 25: 329-334.

Vanapalli S, Fredlund D and Pufah ID (1996) The relationship between the soil-water characteristic curve and the unsaturated shear strength of a compacted glacial till. Geotechnical Testing Journal 19(3): 259-268.
Zazueta FS and Xin J (1994) Soil Moisture Sensors. Florida Cooperative Extension Service, Institute of Food and Agricultural Sciences, University of Florida, Gainesville, FL, USA, Bulletin 229.

\section{WHAT DO YOU THINK?}

To discuss this paper, please submit up to 500 words to the editor at journals@ice.org.uk. Your contribution will be forwarded to the author(s) for a reply and, if considered appropriate by the editorial panel, will be published as a discussion in a future issue of the journal. 